\title{
MANUELS DE FLE ET MAÎTRISE DE LA LANGUE ÉCRITE
} Rebecca CRAVEN'

RÉSUMÉ : Les quatre habiletés de l'enseignement d'une langue étrangère incluent la production écrite $(\mathrm{PE})$ comme une composante indispensable pour la maîtrise de la langue. Cependant, est-ce que la manière dont on la travaille dans les manuels scolaires développe cette capacité ? Selon Courtillon (2003), la présentation d'un modèle d'écriture est nécessaire si on veut éviter la production d'un texte qui suit les structures de la langue maternelle. Cette orientation conduira notre étude du manuel Le Nouveau Taxi ! 1 (Capelle, Menaud, 2009), qui est utilisé couramment dans le cours de français élémentaire (FREN*1150) à l'Université de Guelph et qui affirme mener à la maîtrise de la langue écrite. Pour développer notre recherche: i) nous discuterons de la présentation des tâches écrites et des genres textuels; et à un deuxième niveau, ii) nous analyserons le récit d'expérience et le journal de bord d'une enseignante du cours. Ces données, complétées par les résultats des études antérieures (Schneuwly, Dolz, 1997 et Sales Cordeiro, Michelan de AzeveDO, Prado Mattos, 2000), nous permettront
1 Universidade de Guelph. E-mail: rcraven@uoguelph.ca 
de discuter de la pertinence de l'approche actionnelle présentée dans ce manuel pour la maîtrise de la langue et de comment son utilisation en classe promeut ou pas cette capacité.

MOTS CLÉS : production écrite, modèle d'écriture, genre textuel, manuel de FLE

ABSTRACT : The four skills required in second language teaching include written production as a requirement for the mastery of the language. However, does the way that textbooks present this skill actually develop it? According to Courtillon (2003), the presentation of a written model is required in order to avoid the production of a text that follows the same structure as the maternal language. This approach will guide our evaluation of the textbook Le Nouveau Taxi! 1 (Capelle, Menaud, 2009), which is used in the course Elementary French (FREN*1150) at the University of Guelph and which claims to permit the mastery of the written language. To develop our research: i) we will discuss the presentation of written tasks and textual genres in the manual; and, secondly, ii) we will analyse the reflection and the personal log of the current teacher of this course. This evidence, complemented by results of previous studies on the study of textual genres (Schneuwly, Dolz, 1997 and Sales Cordeiro, Michelande Azevedo,Prado Mattos, 2000), will allow us to discuss the suitability of action-based learning presented in this textbook in regards to language mastery and how its use does or does not promote this ability.

KEY WORDS: written production, writing model, textual genres, FSL textbook 
La production écrite (PE) est une des quatre habiletés indispensables dans la maîtrise d'une langue étrangère (LE). Le genre d'activités proposées pour formuler cette habilité évolue des phrases simples aux histoires complexes écrites par l'apprenant (Cuq, Gruca, 2005). Il existe grosso modo deux catégories générales de la PE : les activités d'écriture et les activités de réécriture (Cuq, Gruca, 2005, pp. 452-456). Les activités de réécriture peuvent être simples, comme une élaboration à partir des points clés d'une histoire, ou un peu plus difficiles, comme le changement d'un genre textuel, par exemple, une lettre, à un autre, comme une narration. De l'autre côté, ce qu'on appelle la réparation du texte, où l'étudiant continue une histoire, et les matrices de texte, où l'étudiant apprend les mécanismes textuels pour écrire une telle PE à travers un modèle, sont tous les deux des exemplaires des activités d'écritures (Cuq, Gruca, 2005, pp. 452-456).

Malgré cette gamme d'activités possibles, il faut travailler la PE d'une façon qui permette effectivement la maîtrise de la langue écrite. Selon Courtillon (2003), une approche didactique qui déchiffre une matrice de texte permet l'enseignement de la PE d'une façon complète. Cette démarche pédagogique, qui s'appelle l'étude des genres textuels, aide l'apprenant à se souvenir des exemplaires d'un tel genre dans la LE étudiée (Courtillon, 2003). Néanmoins, sans l'implication de l'étudiant dans ces activités, il les interprète comme inutiles (Hofhues, Heudorfer, 2013). Pour combattre ce problème, on voit l'influence du Cadre européen commun de référence (CECR) et de l'approche actionnelle sur les genres étudiés (Courtillon, 2003). Les PE travaillées dans le français langue étrangère (FLE) appartiennent aux genres qui exigent l'interaction et la communication (Courtillon, 2003), qui nous renvoient à la définition du verbe « écrire » par Cuq et Gruca (2005) : « écrire, c'est donc produire une communication au moyen d'un texte et c'est aussi écrire un texte dans une langue écrite » (p. 188).

Dans cette étude, nous nous intéressons à comment les manuels scolaires de FLE travaillent la PE et si cette approche mène à la maîtrise des capacités communicatives de la langue écrite. Nous avons abordé cette question en examinant si le manuel scolaire Le Nouveau Taxi ! 1 démontre les critères nécessaires selon notre méthodologie. Finalement, avec l'analyse des activités présentées dans ce manuel, la démarche didactique indiquée et les résultats en salle de classe donnés par une enseignante qui utilise ce manuel à l'Université de Guelph, nous avons tiré des conclusions sur la PE dans ce manuel de FLE. 


\section{Cadre théorique}

Nous commencerons notre examen du Nouveau Taxi ! 1 en présentant les deux assises théoriques nécessaires pour suivre cette étude : l'approche actionnelle et l'enseignement des genres textuels.

\subsection{L'approche actionnelle}

L'avant-propos du Nouveau Taxi ! 1, écrit par ses auteurs, indique qu'ils ont « accentué l'approche actionnelle » dans ce manuel (Capelle, Menard, 2009, p. 3). Avec cette indication évidente, il faut aborder la définition et les implications de cette approche sur l'enseignement du FLE afin d'évaluer le manuel selon des critères pertinents. L'approche actionnelle a eu ses débuts théoriques en améliorant quelques idées déjà proposées par l'approche communicationnelle (Tagliante, 2006). Cette approche didactique propose donc l'utilisation de l'auto-évaluation à propos des tâches que l'étudiant de langue peut faire dans la LE qui le rendent plus engagé dans son apprentissage (Tagliante, 2006). Ces tâches, qui se servent des « composantes linguistique, sociologique et pragmatique» (Rosen, 2006, p. 26), soutiennet l'indépendance de l'apprenant dans la langue cible (LC) pour qu'il puisse être un « acteur social » (Conseil de l'Europe, 2001). Cette définition explique clairement les raisons pour lesquelles un étudiant apprend une langue, qui sont « pour exprimer et dire quelque chose, [alors] les propositions méthodologiques doivent donc s'efforcer d'initier et de motiver une communication réelle en langue étrangère » (Cuq, Gruca, 2005, p. 190). Avec cet enseignement du savoir-agir, l'apprenant est capable d'interagir et de comprendre, à l'oral et à l'écrit, comment survivre dans la vie quotidienne dans un milieu francophone (Tagliante, 2006). Avec cette compréhension de l'approche actionnelle, nous pouvons examiner et interpréter les tâches d'écriture suggérées dans Le Nouveau Taxi ! 1 selon leur vraisemblance aux tâches quotidiennes auxquelles l'apprenant va faire face.

\subsection{L'enseignement des genres textuels}

C'est en suivant l'approche actionnelle appliquée à l'enseignement de la PE qu'on soutient l'intérêt d'enseigner les genres textuels. Cette méthode, détaillée par Courtillon (2003) et Cuq et Gruca (2005), renforce et soutient les exigences du CECR tout d'abord en utilisant les genres textuels quotidiens. Au lieu de donner des tâches scolaires, cette démarche utilise les tâches quotidiennes, où l'apprenant voit 
clairement comment non seulement interpréter les genres textuels qui l'entourent dans la vraie vie, mais aussi comment il faut écrire pour interagir avec un locuteur de la LE. Avec un genre, on ajoute une situation d'écriture, qui, au début, aide l'étudiant à « réaliser un acte de la vie courante » avant de devenir plus abstraite (Cuq, Gruca, 2005, p. 187). L'instance de communication, où l'objectif est d'écrire pour communiquer, est absolument nécessaire dans cette approche ainsi que la production personnelle de l'étudiant (Courtillon, 2003). La démarche didactique de cette approche suit plusieurs étapes.

Cuq et Gruca (2005) et Courtillon (2003) expliquent comment établir ce processus d'écriture, qui exige l'enseignement de la compréhension des textes dans la LE avant que l'apprenant commence à écrire. Il existe trois étapes dans ce processus : 1) établir le contexte ; 2) établir une mémoire des connaissances ; et 3) écrire la PE. La première étape sert à déchiffrer un modèle, qu'il soit authentique ou pédagogique, du genre textuel que l'étudiant écrira à la fin. Il faut guider l'apprenant dans sa compréhension de cette matrice de texte pour établir les consignes du genre, ce qu'on appelle l'enseignement « global » du modèle (Cuq, Gruca, 2005, p. 187). A travers la compréhension écrite d'un texte appartenant au genre étudié, l'apprenant doit découvrir luimême, en répondant aux questions ciblées de l'enseignant, l'objectif du texte, comment on établit cet objectif, qui l'a écrit, qui est l'audience ciblée, le temps verbal utilisé et les autres règles qui s'appliquent au genre, comme dans les ateliers d'écriture proposés par Dolz en 1998 sur le récit d'énigme. Après avoir étudié le genre textuel, l'enseignant doit attirer l'attention sur les connaissances antérieures que l'étudiant possède ou, également, enseigner des nouvelles connaissances nécessaires pour produire une PE de ce genre. Par exemple, avec un certain sujet pour la $\mathrm{PE}$, comme donner des indications à un ami pour se rendre quelque part, il faut premièrement établir un vocabulaire et les capacités linguistiques pour accomplir la tâche, par exemple, il faut travailler l'impératif avant d'être capable de donner des indications (Cuq, Gruca, 2005). Finalement, quand on passe au processus de production, il existe trois sous-étapes : la planification, la mise en texte et la révision (Cuq, Gruca, 2005, p. 185). Ce processus dans son entièreté soutient l'acquisition des « savoir-faire écrits » voulus (Courtillon, 2003, p. 74) en soulignant les processus mentaux qui se passent en écrivant (Cuq, Gruca, 2005, p. 186).

Les études antérieures dans ce domaine renforcent ces conclusions. En 2000, Sales Cordeiro, Michelan de Azevedo et Prado Mattos ont effectué une étude sur l'écriture du récit d'aventure. Ils ont commencé tout d'abord avec l'instruction simple aux étudiants pour écrire 
une histoire d'aventure ; après, ils ont organisé l'étude du genre par des ateliers pertinents. Les deux élèves de l'étude ont tous les deux amélioré leur capacité d'écrire ; les deux histoires étaient plus cohérentes et ont suivi les consignes du genre (Sales Cordeiro, Michelan de Azevedo, Prado Mattos, 2000). Étant donné ces bases théoriques et pratiques, nous prendrons, dans cette étude, l'utilisation de cette séquence didactique comme indicateur que l'activité doit mener à la maîtrise de la langue écrite, si elle garde les objectifs communicatifs démontrés par l'approche actionnelle.

\section{Méthodologie}

Dans cette étude, nous évaluerons les PE détaillées dans Le Nouveau Taxi! 1 selon les exigences de l'objectif communicatif de l'approche actionnelle et des trois étapes nécessaires de la séquence didactique des genres textuels. Néanmoins, il faut tout d'abord établir le contexte de cette recherche en expliquant l'utilisation du Nouveau Taxi ! 1 à l'Université de Guelph et comment nous évaluerons les activités de PE présentées par ce manuel et le cours de FREN*1150.

\subsection{Contexte}

Nous utilisons Le Nouveau Taxi ! 1 à l'Université de Guelph dans l'enseignement du cours FREN*1150 : le français élémentaire. Ce cours est ouvert aux étudiants qui ont leur onzième année de français cadre ou l'équivalent selon les consignes du curriculum ontarien (University of Guelph, 2014). Nous présumons qu'ils ont tous les habiletés délimitées par le curriculum ontarien au niveau des quatre habiletés langagières (Ontario, 2000). Néanmoins, le nombre d'années qui ont passé depuis cette onzième année d'école secondaire est très variable, comme l'enseignante indique dans son récit d'expérience (Craven, $2014 \mathrm{a}^{2}$ ). En somme, ce cours a le même niveau de leur douzième année d'école secondaire et offre aux étudiants une base pour continuer leur apprentissage du français au niveau universitaire. Les étudiants de la maîtrise en études françaises prennent le rôle de l'enseignant et effectuent ce cours sous la direction d'un coordinateur, un professeur du département d'études françaises à l'Université de Guelph. Le cours dure deux heures cinquante minutes chaque mardi soir (UNIVERSITY OF GUELPH, 2014).

Nous suivons le manuel Le Nouveau Taxi! 1 dans l'enseignement de ce cours ; le manuel et son cahier d'activités sont les livres

2 Le récit d'expérience complet est disponible pour consultation dans les annexes (Annexe 5.2 
exigés sur le plan du cours que les étudiants doivent apporter en classe chaque jour (UNIVERSITY OF GUELPH, 2014). Nous aimerions souligner également que Le Nouveau Taxi ! 1 se base sur les niveaux du CE$\mathrm{CR}$ et correspond aux exigences du niveau A1. Dans cette étude, nous nous focaliserons sur l'audience envisagée par la tâche écrite, la vraisemblance de l'activité comme tâche personnelle et la présence (ou pas) d'une séquence didactique pour travailler le genre textuel en PE.

\subsection{Données}

Pour examiner si Le Nouveau Taxi ! 1 soutient la maîtrise de la PE chez les apprenants de $\mathrm{FLS}^{3}$, nous étudierons trois données : le manuel lui-même, le journal de bord (Craven, 2014b) et le récit d'expérience (Annexe 5.2) de l'enseignant qui a enseigné ce cours en automne 2013. Avec le manuel comme guide, nous étudierons si les exercices délimités comme des activités qui travaillent l'écrit suivent la présentation de la PE selon l'enseignement des genres textuels (Courtillon, 2003) et selon la notion des tâches quotidiennes soulignées par le CECR (Conseil de l'Europe, 2001). Nous noterons le succès d'une activité particulière dans notre sélection des PE selon les cadres théoriques de la séquence pédagogique suggérée et le genre produit, où nous attendions que les étudiants écrivent « en situation de production personnelle » (Courtillon, 2003 p. 112). Si l'interlocuteur n'était pas déterminé dans la consigne de l'activité, nous avons considéré que le travail n'aurait pas d'objectif communicatif. Notre deuxième niveau de sélection était la séquence didactique des genres textuels, que les anciennes études ont déjà démontrée comme capable de mener à la maîtrise de la langue écrite (Sales Cordeiro, Michelan de Azevedo, Prado Mattos, 2000). Notre dernière sélection était au niveau de l'implication de l'apprenant ; est-ce que la tâche était personnelle ou pas?

Nous avons établi les PE que nous étudierions à travers la légende du manuel ; il identifie toutes les PE avec l'image d'un stylo à côté des instructions, ou à travers le titre «Production Écrite »si l'activité se trouve dans les chapitres d'évaluation. On n'a pas examiné les exercices qui travaillaient la PE après l'unité sept du Nouveau Taxi ! 1, parce que le cours de FREN*1150 (UNIVERSITY OF GUELPH, 2014) n'aborde que les sept premières unités du manuel. Nous avons évalué trois autres activités de PE à part du manuel aussi en étudiant le récit d'expérience (CRAVEN, 2014a) et le journal de bord (CRAVEN, 2014b ${ }^{4}$ ) de l'enseignante de ce cours lors des deux derniers semestres.

\section{Français Langue Second}

4 Le journal de bord complet est disponible pour consultation dans les annexes (Annexe 5.3) 


\section{Analyse}

Pour commencer notre analyse, il a fallu évaluer tout d'abord les exercices présentés dans Le Nouveau Taxi ! 1. Par la suite, le journal de bord et le récit d'expérience de l'enseignante ont permis d'ajouter des informations sur la manières dont l'enseignante avait traité la PE en salle de classe selon ou malgré les intentions du manuel.

\subsection{Manuel}

Nous avons identifié vingt-deux instances de PE dans les sept unités premières du Nouveau Taxi! 1 selon nos exigences déjà indiquées. Nous résumons chaque activité scolaire selon trois catégories, l'audience, la séquence didactique et l'implication de l'étudiant, dans le Tableau 1, qui se trouve dans l'Annexe 5.1. En commençant avec notre premier niveau de sélection, nous avons éliminé les activités « Poème, » «Une journée avec, » « Souvenirs, souvenirs, » "Comment réussir... , » et " Petit guide, » parce qu'il n'y avait pas d'interlocuteur. Le manque d'objectif communicatif était surprenant, spécialement en considérant la nature actionnelle du manuel.

La prochaine sélection se basait sur la présence de la séquence didactique souhaitée : l'enseignement du genre textuel. Nous avons gardé « Votre e-mail, » « Carte postale, » « Habitudes, » «Une belle fête, » « Règlement intérieur, » « Le temps de vacances, » et « Les vacances dans votre pays. » Ces activités ont partagé l'étude d'une matrice de texte ; nous avons ajouté le genre des instructions qu'on peut observer dans le manuel dans le Tableau 1.

Finalement, nous avons identifié les activités qui ont suivi toutes nos exigences. Si l'étudiant a dû réfléchir, tirer des conclusions à partir des informations présentées, convaincre le lecteur de son travail ou présenter ses propres idées, nous avons gardé l'activité ; la tâche d'écriture était personnelle. Après cette sélection, seulement cinq des vingt-deux activités ont été conservées : « Carte postale, » « Demande d'informations, » Habitudes alimentaires, » «Une belle fête, » et « Règlement intérieur. » (Annexe 5.1, Tableau 1). Selon nos critères et exigences, ces cinq PE sont les seules qui proposent une activité complète, pouvant mener effectivement à la maîtrise de la langue écrite dans Le Nouveau Taxi ! 1. 


\subsection{Journal de bord et récit d'expérience}

Malgré les résultats que nous avons trouvés en étudiant $L e$ Nouveau Taxi ! 1, il fallait aussi évaluer si les activités écrites ont été présentées de la même manière et si d'autres activités ont été utilisées pour travailler la PE en salle de classe. Dans le Tableau 2, dans l'Annexe 5.1, nous résumons les détails pertinents des trois PE évaluées pendant le cours de FREN*1150. Nous avons suivi notre évaluation de la même manière qu'avant, donc, nous avons commencé en déterminant l'audience de la PE. La première tâche, avec une correspondante imaginaire, n'était qu'une façon pour l'enseignant de déterminer le niveau de l'apprenant (CRAVEN, 2014a); nous l'avons exclue. Le destinataire de la deuxième tâche, les amis sur Facebook, semblait plus réaliste. L'enseignante ajoute : «Je pense qu'ils se sont identifiés avec la situation donnée » (CRAVEN, 2014b); nous l'avons gardée pour la prochaine sélection. La dernière tâche écrite était d'écrire un article à partir d'une image insolite. Il n'y avait pas d'interlocuteur donné aux élèves (CRAVEN, 2014a), alors nous avons dû éliminer cette activité aussi.

Après cette première sélection, avec seulement la tâche des indications sur Facebook comme vainqueur, nous avons examiné si cette tâche a utilisé notre démarche didactique. Néanmoins, malgré le fait que l'enseignante a travaillé les aspects linguistiques, l'impératif (CRAVEN, 2014b), l'étude d'une matrice de texte n'était pas effectuée. L’enseignante a ajouté dans son rapport d'expérience qu'« On a imaginé que l'impact de Facebook sur leur vie quotidienne était assez fort de (sic.) ne pas inclure un modèle pour la deuxième production écrite » (CRAVEN, 2014a). Par contre, avec l'implication des étudiants envers cette tâche (CRAVEN, 2014b), cette PE aurait réussi notre troisième niveau de sélection.

Curieusement, l'enseignante a utilisé cette démarche pour les deux autres PE, au moins pendant son premier semestre (CRAVEN, 2014a). Pour la première PE en automne 2013, elle a utilisé une matrice de texte tirée du Nouveau Taxi ! 1 (CRAVEN, 2014a). Cependant, l'objectif de cette PE était de voir le niveau de l'étudiant pour voir si l'apprenant était sur qualifié ou sous qualifié pour le cours (CRAVEN, 2014a). Avec la matrice de texte, l'enseignante note que :

Cependant, après avoir reçu une vingtaine des courriels, qui commençaient tous avec "Cher » ou « Chère » ami, qui parlaient des mêmes trucs quotidiens et qui se terminaient avec «À plus, » j'ai compris, enfin, que le déchiffrement de modèle n’a pas permis l'objectif de l'activité. Je 
me suis trouvée incapable de voir si les étudiants étaient trop forts pour le cours. (CRAVEN, 2014a)

Alors, l'utilisation de notre approche souhaitée n'était pas le meilleur choix de séquence didactique à la PE dans ce cas. L'enseignante a modifié l'activité pendant son deuxième semestre, avec les fins positives au niveau de ses buts pour déterminer l'hétérogénéité de sa classe sans l'utilisation de notre approche didactique (CRAVEN, 2014b). La dernière PE a aussi utilisé l'approche de l'enseignement de la langue écrite par les genres textuels (CRAVEN, 2014a).

De plus, pour la deuxième et la troisième $\mathrm{PE}$, l'objectif langagier des activités était de maîtriser un temps verbal. Par exemple, l'enseignante ajoute que " Nous avons beaucoup travaillé l'impératif aujourd'hui. Je pense que c'est maintenant clair pour eux, spécialement en regardant leurs productions écrites ce soir »(CRAVEN, 2014b). L'aspect communicatif de la langue reste une faiblesse avec la maîtrise purement grammaticale de la langue, comme Schneuwly et Dolz (1997) ont prévu. Il semble que l'enseignant et le manuel scolaire essayent de suivre l'approche détaillée par Courtillon (2003) en proposant d'étudier de manière approfondie un texte pour que l'étudiant ait des exemplaires dans la LE d'un tel genre textuel, mais l'objectif de la $\mathrm{PE}$, la communication, n'est pas impliquée.

\section{Conclusion}

Le Nouveau Taxi ! 1 applique et renforce l'utilisation d'une matrice de texte en guidant la PE, avec les instructions comme « Selon le modèle » et « Écrire à la manière de. » Néanmoins, ce que nous avons trouvé à travers notre analyse des PE, c'est qu'il y existe un manque de l'implication de l'apprenant à travers l'absence d'objectifs communicatifs dans plusieurs tâches. Nous voyons que malgré l'utilité de cette approche pour maîtriser un genre textuel, il ne faut pas oublier l'importance de l'expression personnelle de chaque étudiant en leur donnant l'occasion d'écrire. Seulement cinq activités sur les vingt-deux présentés dans les sept unités premières du Nouveau Taxi 1 ! ont respecté nos critères de sélection des PE à cause de cette exigence. De la même façon, la PE en salle de classe de FREN*1150 a aussi utilisé la matrice d'un texte, mais, pour deux des trois PE détaillées, ces tâches n'étaient pas communicatives, une nécessité selon le CECR (Conseil de l'Europe, 2001) et selon les experts dans ce domaine (par exemple, Cuq, Gruca, 2005 et Courtillon, 2003). De 
plus, la seule instance où on a vu l'implication de l'étudiant concernant les PE faites en salle de classe, c'était lorsqu'ils n'avaient pas utilisé une matrice de texte.

Ce problème nous renvoie à l'étude de Schneuwly et Dolz (1998) qui a défendu que le désir de maîtriser un genre textuel écrase la communication de la langue écrite. Étant donné nos résultats, nous concluons que le manuel scolaire peut mener à la maîtrise de la PE avec quelques activités d'écriture qu'il propose, selon notre analyse cinq instances sur vingt-deux. Cependant, il faut modifier la plupart des PE en utilisant la séquence didactique des genres textuels pour achever une instance de communication vraisemblable et des objectifs qui impliquent l'étudiant d'une façon personnelle dans son écriture. Nous suggérons que les études futures travaillent la présence de vraies instances communicatives dans la PE chez l'apprenant, parce que la présence forte des PE avec seulement l'objectif de démontrer la compréhension d'un aspect grammatical ne suit pas l'objectif de l'apprenant d'une LE : d'agir dans le monde en communiquant (Rosen, 2006).

\section{Annexes}

Les matériaux cités pendant l'article, créés par l'auteure de cette étude ou par l'Université de Guelph, comme les tableaux, le récit d'expérience, le journal de bord et le plan du cours, peuvent se trouver ici dans les annexes. Annexe 5.1 contient les tableaux; le récit d'expérience se trouve dans l'Annexe 5.2 ; et le journal de bord est dans l'Annexe 5.3.

\section{Annexe 5.1 : Tableaux}

Tableau 1 : L'explication de la classification des PE présentées dans Le Nouveau Taxi ! 1. Les pages indiquées font référence à où chaque activité se trouve dans le manuel.

\begin{tabular}{|l|l|l|l|l|}
\hline Activité & Objectiflangagier & Audience & $\begin{array}{l}\text { Séquence didactique } \\
\text { idéale? }\end{array}$ & Tâche personnelle ? \\
\hline $\begin{array}{l}\text { Votre e-mail en } \\
\text { français }(p .21)\end{array}$ & Se présenter & $\begin{array}{l}\text { Correspondant } \\
\text { imaginaire }\end{array}$ & Oui (« Comme Marco ») & Non \\
\hline $\begin{array}{l}\text { Fiche des } \\
\text { détails }(p .22)\end{array}$ & Se présenter & Autres étudiants & Non & Non \\
\hline $\begin{array}{l}\text { Présentez une } \\
\text { amie }(p .22)\end{array}$ & $\begin{array}{l}\text { Présenter quelqu'un } \\
\text { d'autre }\end{array}$ & $\begin{array}{l}\text { Correspondant } \\
\text { imaginaire }\end{array}$ & Non & Non \\
\hline
\end{tabular}




\begin{tabular}{|c|c|c|c|c|}
\hline Poème (p. 31) & Écrire un poème & Personne & $\begin{array}{l}\text { Oui («À la } \\
\text { manière de ») }\end{array}$ & Non \\
\hline Ma chambre (p. 32) & Décrire votre chambre & $\begin{array}{l}\text { Correspondant } \\
\text { imaginaire }\end{array}$ & Non & Non \\
\hline $\begin{array}{l}\text { Carte postale } \\
(\text { p. } 41)\end{array}$ & Décrire Marseille & $\begin{array}{l}\text { Correspondant } \\
\text { imaginaire }\end{array}$ & $\begin{array}{l}\text { Oui (« Lisez la carte } \\
\text { postale d'Audrey. } \\
\text { À votre tour ») }\end{array}$ & $\begin{array}{l}\text { Oui (interprétation } \\
\text { des faits) }\end{array}$ \\
\hline $\begin{array}{l}\text { Écrire un message } \\
\text { simple ( } p .44)\end{array}$ & $\begin{array}{l}\text { Suggérer un } \\
\text { restaurant à Sébastien }\end{array}$ & $\begin{array}{l}\text { Sébastien } \\
\text { (personnage fictif) }\end{array}$ & Non (évaluation) & $\begin{array}{l}\text { Oui (interprétation } \\
\text { des faits) }\end{array}$ \\
\hline $\begin{array}{l}\text { Une journée } \\
\text { avec (p. } 53)\end{array}$ & $\begin{array}{l}\text { Décrire la journée } \\
\text { d'un ami }\end{array}$ & Personne & $\begin{array}{l}\text { Oui («À la manière } \\
\text { de l'article ») }\end{array}$ & Non \\
\hline Invitation ( $p .54)$ & Refusez une invitation & $\begin{array}{l}\text { Paul (personnage } \\
\text { fictif) }\end{array}$ & Non & Non \\
\hline $\begin{array}{l}\text { Demande } \\
\text { d'informations } \\
\text { (p. 54) }\end{array}$ & $\begin{array}{l}\text { Donner des } \\
\text { informations sur } \\
\text { un club de sport }\end{array}$ & $\begin{array}{l}\text { Correspondant } \\
\text { imaginaire }\end{array}$ & Non & $\begin{array}{l}\text { Oui (interprétation } \\
\text { des faits) }\end{array}$ \\
\hline $\begin{array}{l}\text { Souvenirs, } \\
\text { souvenirs ( } p .63)\end{array}$ & $\begin{array}{l}\text { Raconter un } \\
\text { souvenir de fête }\end{array}$ & Personne & $\begin{array}{l}\text { Oui (suite des activités } \\
\text { dans la leçon 20) }\end{array}$ & Non \\
\hline $\begin{array}{l}\text { Habitudes } \\
\text { alimentaires (p. 64) }\end{array}$ & $\begin{array}{l}\text { Écrire les habitudes } \\
\text { alimentaires de } \\
\text { votre pays }\end{array}$ & $\begin{array}{l}\text { Le magazine Elle à } \\
\text { Table et son public }\end{array}$ & $\begin{array}{l}\text { Oui (« Aidez-vous du } \\
\text { modèle suivant ») }\end{array}$ & $\begin{array}{l}\text { Oui (implication } \\
\text { de l'étudiant en } \\
\text { expliquant ses } \\
\text { habitudes) }\end{array}$ \\
\hline $\begin{array}{l}\text { Une belle fête } \\
(p .64)\end{array}$ & Raconter une fête & $\begin{array}{l}\text { Correspondant } \\
\text { imaginaire }\end{array}$ & $\begin{array}{l}\text { Oui (question de } \\
\text { planification) }\end{array}$ & $\begin{array}{l}\text { Oui (implication } \\
\text { de l'étudiant en } \\
\text { expliquant sa vie) }\end{array}$ \\
\hline $\begin{array}{l}\text { Comment } \\
\text { réussir... (p. } 73)\end{array}$ & Écrire un petit guide & Personne & $\begin{array}{l}\text { Oui («À la } \\
\text { manière de ») }\end{array}$ & Non \\
\hline $\begin{array}{l}\text { Règlement } \\
\text { intérieur ( } p .74)\end{array}$ & Faire des propositions & Directeur de l'école & $\begin{array}{l}\text { Oui (image d'une } \\
\text { liste et un exemple) }\end{array}$ & $\begin{array}{l}\text { Oui (pensée critique } \\
\text { de ce qui va améliorer } \\
\text { sa vie à l'école) }\end{array}$ \\
\hline $\begin{array}{l}\text { Fête d'anniversaire } \\
(p .74)\end{array}$ & Donner des conseils & $\begin{array}{l}\text { Théo (personnage } \\
\text { fictif) }\end{array}$ & Non & Non \\
\hline Petit guide (p.74) & Donner des conseils & Personne & $\begin{array}{l}\text { Oui («Sur le modèle } \\
\text { de Comment réussir } \\
\text { un entretien ») }\end{array}$ & Non \\
\hline $\begin{array}{l}\text { Parler } \\
\text { d'événements } \\
\text { passés (p. } 76)\end{array}$ & $\begin{array}{l}\text { Raconter les } \\
\text { derniers vacances }\end{array}$ & $\begin{array}{l}\text { Correspondant } \\
\text { imaginaire }\end{array}$ & Non & Non \\
\hline $\begin{array}{l}\text { Refuser une } \\
\text { proposition (p. } 76)\end{array}$ & $\begin{array}{l}\text { Répondre à une } \\
\text { invitation au négatif }\end{array}$ & $\begin{array}{l}\text { Fabien (personnage } \\
\text { fictif) }\end{array}$ & Non & Non \\
\hline Portrait (p. 85) & $\begin{array}{l}\text { Décrire les vacances } \\
\text { de quelqu'un d'autre }\end{array}$ & Autre étudiant & Non & Non \\
\hline $\begin{array}{l}\text { Le temps de } \\
\text { vacances (p. } 86)\end{array}$ & $\begin{array}{l}\text { Répondre à un e-mail } \\
\text { à propos des vacances }\end{array}$ & $\begin{array}{l}\text { Adriana (personnage } \\
\text { fictif) }\end{array}$ & $\begin{array}{l}\text { Oui (l'e-mail d'Adriana } \\
\text { fournit un exemplaire) }\end{array}$ & Non \\
\hline $\begin{array}{l}\text { Les vacances dans } \\
\text { votre pays }(p .86)\end{array}$ & $\begin{array}{l}\text { Décrire les habitudes } \\
\text { des vacances }\end{array}$ & $\begin{array}{l}\text { Pour le public d'un } \\
\text { magazine français }\end{array}$ & $\begin{array}{l}\text { Oui (« Sur le modèle } \\
\text { de la page } 84 »)\end{array}$ & Non \\
\hline
\end{tabular}

Tableau 2 : L'explication de chaque PE notée dans le cours de FREN*1150. On a résumé ces descriptions à partir du récit d'expé- 
rience (Annexe 5.2) et du journal de bord (Annexe 5.3). *Changement pour hiver 2014 (auparavant, c'était « Oui »; Annexe 5.2).

\begin{tabular}{|l|l|l|l|}
\hline & Production 1 & Production 2 & Production 3 \\
\hline Objectif & $\begin{array}{l}\text { Apprendre le niveau } \\
\text { de l'apprenant }\end{array}$ & $\begin{array}{l}\text { Utiliser l'impératif et les } \\
\text { prépositions du lieu }\end{array}$ & $\begin{array}{l}\text { Utiliser le passé composé } \\
\text { et le futur proche }\end{array}$ \\
\hline $\begin{array}{l}\text { Description } \\
\text { de l'activité }\end{array}$ & $\begin{array}{l}\text { Écrire un courriel à un } \\
\text { correspondant français } \\
\text { où vous vous présentez. }\end{array}$ & $\begin{array}{l}\text { Donner des indications à vos } \\
\text { amis pour venir à votre fête } \\
\text { dans un message Facebook. } \\
\text { Commencer à l'Université. }\end{array}$ & $\begin{array}{l}\text { A partir d'une image insolite, } \\
\text { imaginer les événements (les } \\
\text { accents) qui viennent de se passer } \\
\text { et ce qui vont se passer. }\end{array}$ \\
\hline $\begin{array}{l}\text { Utilisation d'un } \\
\text { modèle concret }\end{array}$ & Non* & Non & Oui \\
\hline
\end{tabular}

\section{Annexe 5.2 : Récit d'expérience ${ }^{5}$ : Rebecca Craven, Au- tomne 2013}

Je veux commencer en disant que mon expérience comme assistante de langue n'était pas ce que j'ai attendu lorsque le premier rencontre de l'équipe du cours, FREN*1150 : le français élémentaire. Avec mes idées à la fois ambitieuses et naïves, comme « tout le monde aimera le français après mon cour» j'étais fortement déçue quand notre coordinatrice nous a dit que la plupart des étudiants ne poursuivraient pas de cours en français. Mes illusions étaient brisées, mais, au cours du semestre, j'ai appris que les étudiants voulaient prendre le français pour des raisons admirables.

Le premier cours était assez facile, mais quand même c'étaient trois heures de ma vie que je ne peux pas vraiment me rappeler aujourd'hui, parce que c'était si rapide! J'adorais mes « enfants, » spécialement les deux « adultes, » un homme et une femme de 40 ans qui, tous les deux, ont pris le cours avec les espoirs ambitieux. L'homme voudrait devenir enseignant de français et la femme n'est jamais allée à l'université, alors c'était son premier cours universitaire! J'ai appris que le stéréotype, que ces étudiants n'iraient pas continuer le français après ce cours, était peut-être vrai pour quelques étudiants, mais ils ont tous partagé un désir d'apprendre le français.

Malgré cela, j’ai continué à ressentir quelques doutes pendant la première production écrite. Comme le cours se passe vite avec douze classes, douze semaines, trois heures par classe, il fallait avoir des évaluations presque chaque soirée. Je l'ai trouvé un peu injuste envers l'apprenant : il fallait apprendre pendant deux heures, et puis après, sans avoir l'occasion d'étudier, l'étudiant devait produire des productions écrites ou orales pour être évalué. En planifiant la première production

\section{Le récit est présenté tel qu'il a été écrit, sans correction linguistique.}


écrite, étant donné que nous n'avons pas d'indice à propos du niveau de l'apprenant sauf ce qu'ils ont écrit sur leur formulaire d'inscription, cette activité nous donnerait l'occasion de voir leurs compétences.

La démarche était, à la fin du cours, sans instructions, de leur demander d'écrire un courriel à un correspondant français. Néanmoins, à mon avis, il fallait leur fournir un modèle. On a discuté en classe comment se présenter, mais l'importance des salutations et des terminaisons d'une lettre n'était pas enseignée. Alors, nous avons utilisé un modèle, trouvé dans la leçon 4 dans Le Nouveau Taxi ! 1 de Hachette (2009). Cependant, après avoir reçu une vingtaine des courriels, qui commençaient tous avec « Cher » ou « Chère » ami, qui parlaient des mêmes trucs quotidiens et qui se terminaient avec « À plus, » j'ai compris, enfin, que le déchiffrement de modèle n'a pas permis l'objectif de l'activité. Je me suis trouvée incapable de voir si les étudiants étaient trop forts pour le cours.

Pour les autres productions écrites, les indications pour se rendre à une fête sur Facebook et l'histoire qui entourait une image insolite, les étudiants ont travaillé en équipes. J’ai eu la capacité de voir, de cette manière, qui était l'étudiant le plus fort à l'écrit dans un groupe de deux ou trois, qui a révisé le travail avant de le remettre, qui a proposé les idées plus complexes, etc. Ils ont aussi eu l'expérience d'apprendre à travers leurs paires de cette manière. On a imaginé que l'impact de Facebook sur leur vie quotidienne était assez fort de ne pas inclure un modèle pour la deuxième production écrite, mais pour la troisième, on a déchiffré un document scolaire que l'ancienne enseignante a créé afin de voir l'utilisation des temps verbaux du passé composé et du futur simple.

Après cet événement, je me suis rendu compte que j'étais toujours étudiante moi-même. Après cette réalisation, la préparation pour chaque cours avec ma coordinatrice et l'assistante de langue qui l'a enseigné l'année dernière était bien passée. Dans la salle de classe, après avoir parlé avec mes élèves, j'ai compris leurs buts en suivant ce cours. C'était une occasion pour eux de pratiquer, de ne pas perdre la langue qu'ils ont apprise à l'école, de se rappeler même si le cours ne compterait pour rien qu'un électif. Ils voudraient voyager en France et au Québec, parler avec leurs nouveaux amis à l'université qui sont francophones, etc. Ce n'était pas une perte s'ils ne suivraient pas le prochain cours, FREN"1200.

Cette expérience, malgré ses débuts inquiétants, m’a donnée l'occasion de comprendre comment et pourquoi nous enseignons la langue française. Au fond, il faut permettre à des apprenants d'être cu- 
rieux, de promouvoir leurs capacités de communiquer et d'explorer leur monde et, finalement, de leur permettre de pratiquer leurs habiletés dans une atmosphère sûre et motivante.

\section{Annexe 5.3 : Journal de bord : Rebecca Craven, Hiver 2014}

Le 6 janvier, 2014

Les étudiants sont un peu partout ce semestre. La moitié est trop forte, l'autre moitié trop faible. J'ai recommandé à quelques étudiants de prendre le cours prochaine ou le cours en ligne. Malgré cela, la classe semble motivée et tout le monde a signé notre contrat pour le semestre!

\section{Le 13 janvier, 2014}

Ce semestre, j'ai présenté la première évaluation écrite sans l'étude d'un courriel français. Je pense que c'était un peu stressant pour quelques étudiants, mais la majorité l'a fini dans 15 minutes. En tout cas, il y a eu au moins trois ou quatre étudiants (de vingt-deux) qui n'ont pas ajouté leur nom ou une formule de politesse à la fin; seulement deux ou trois ont utilisé « cher » au début et la plupart ont utilisé « bonjour » ou même « salut. » Ils sont une classe vraiment mixte ce semestre!

\section{Le 20 janvier, 2014}

Pas de questions à propos des courriels aujourd'hui; j’ai réaffirmé « je suis étudiante » au lieu de « je suis une étudiante » et l'orthographe des mots qui ont le son «é » quelque part. Les productions orales étaient bien faites à la fin, sauf que nous n'avons pas eu beaucoup de temps!

Le 27 janvier, 2014

Nous avons eu le premier quiz aujourd'hui à la fin du cours. Pour la plupart, c'était bien réussi. Les étudiants ont créé leurs propres questions pour toute la classe comme activité de révision. Autres étudiants ont corrigé la grammaire de ces questions, puis toute la classe les ont faits ensemble. Ils ont souligné les aspects les plus importants dans notre cours! Ils se sont amusés en pratiquant leur production orale et leur compréhension orale avec l'activité « dessiner la chambre. »

\section{Le 3 février, 2014}

Nous avons beaucoup travaillé l'impératif aujourd'hui. Je pense que c'est maintenant clair pour eux, spécialement en regardant leurs productions écrites ce soir. Ils ont dû écrire les indications à leurs amis 
dans un message Facebook pour trouver leur fête. Sauf qu'un groupe, qui n’a pas utilisé le pronom « on » ni les possessifs dont on a parlés aujourd'hui, c'était bien réussi. Je pense qu'ils se sont identifiés avec la situation donnée, aussi. Ils ont même commencé et terminé leurs messages avec les salutations et les terminaisons correctes, et les fêtes proposées étaient vraiment drôles!

Le 10 février, 2014

Il y avait un manque d'énergie ce soir. Nous avons commencé avec un jeu de retrouvailles, mais les étudiants n'ont pas vraiment participé. Par contre, ils ont rapidement compris les niveaux de langue et comment poser les questions différemment. Pour la production orale, nous avons eu des entretiens d'un talk-show avec les personnages connus. À la fin de chaque présentation, les autres étudiants ont dû deviner le personnage selon les informations données de leur quotidien. C'était vraiment drôle et bien réussi!

\section{Le 24 février, 2014}

C'était le deuxième quiz aujourd'hui. Comme d'habitude, on a commencé avec les exercices de révision : un genre de BINGO, un remue-ménage des verbes utilisés pour indiquer un chemin et les prépositions de lieu, et l'activité « Fixer rendez-vous » où ils ont travaillé leur expression orale. Ce quiz était le pire le semestre dernier, mais ce semestre, ce n'était pas si mal.

\section{Le 4 mars, 2014}

Cette semaine, on n'a pas eu beaucoup de temps à la fin du cours (même si on a annulé la pause pour qu'ils puissent partir tôt)! Ils ont aimé le jeu de pictionnaire et je pense qu'ils ont compris les expressions de quantités et l'article partitif. Comme le semestre dernier, la présentation du passé composé était un succès! C'était évident avec leurs productions orales qu'ils ont compris comment le formuler avec être ou avoir.

\section{RÉFÉRENCES BIBLIOGRAPHIQUES:}

Capelle, G., \& Menaud, R. Le Nouveau Taxi ! 1. Paris: Hachette Éducation, 2009.

Conseil de l'Europe. Le Cadre européen commun de référence. Strasbourg : Didier, 2001. 
Courtillon, J. Élaborer un cours de FLE. Paris: Hachette Livre, 2003.

Craven, R. Journal du cours: FREN*1150 hiver 2014.Non publié, 2014.

Craven, R. Récit d'expérience: FREN*1150 automne 2013. Non publié, 2014.

Cuq, J.-P., \& Gruca, I. Cours de didactique du français langue étrangère et seconde. Grenoble: Presses universitaires de Grenoble, 2005.

Dolz, J. Apprendre le français avec des récits d'énigme. Genève : FPSE, Université de Genève, 1998.

Hofhues, S., \& Heudorfer, A. «Students' Publishing Projects and their Impact on Teaching and Learning ». Dans : M. Patrut et B. Patrut (Éds.), Social Media in Higher Education : Teaching in Web 2.0 (179-196). Hershey : Information Science Reference, 2013.

Ontario Ministry of Education. The Ontario Curriculum Grades 11 and 12: French as a Second Language-Core, Extended, and Immersion French. Toronto: Ontario Ministry of Education, 2000.

Rosen, É. Le point sur le Cadre européen commun de référence pour les langues. Lassay-les-Château : CLE International, 2006.

Sales Cordeiro, G., Michelan De Azevedo, I. C., \& Prado Mattos, V. L. Écrire un récit d'aventures à l'école et à la clinique logopédique. Revue Tranel, 33, 183-197, 2000.

Schnewly, B., \& Dolz, J. « Les genres scolaires, des pratiques langagières aux objets d'enseignement ». Repères. Lyon v. 15, n. 1, p. 167185, 1997.

University of Guelph. Course outline : FREN*1150 Elementary French. Guelph : University of Guelph, 2014. 